\title{
POŚTA
}

TELEKOMUNIKÁCIE A

ELEKTRONICKÝ OBCHOD

\section{KUNDENZUFRIEDENHEIT ALS UNTERNEHMENSZIEL}

\author{
Thomas Bader $^{1}$ - Iveta Kremenova ${ }^{2}$
}

\section{Ausgangssituation}

Zunehmende Globalisierung der Märkte und der Umgang mit immer kritischeren, weniger loyalen Kunden stellt neben der Qualität der erbrachten Leistungen von Unternehmen, die Kundenzufriedenheit als den wettbewerbsdifferenzierenden Erfolgsfaktor dar.

Darum gilt es für die Unternehmen neben der Erbringung hoher Produkt- und Dienstleistungsqualität auch ein funktionierendes Zufriedenheitsmanagement im Unternehmen zu etablieren.

Mit Zufriedenheitsmanagement werden dabei alle Maßnahmen bezeichnet, welche ein Unternehmen ergreift, um Kundenzufriedenheitsziele $\mathrm{zu}$ planen, die Zufriedenheit der Kunden zu messen und zu analysieren, die Ergebnisse innerbetrieblich zu kommunizieren und die Daten so zu nutzen, dass eine Erreichung der Zufriedenheitsziele möglich wird.

Neben der Messung und Aufbereitung der Zufriedenheitsinformationen stellt somit die Nutzung der bereitgestellten Daten einen wesentlichen Teil des Zufriedenheitsmanagements dar. Zufriedenheitsdaten können im Rahmen des Qualitätsmanagements zur Sicherung und Verbesserung der angebotenen Produkte und Dienstleistungen genutzt werden und / oder zur Steuerung des Verhaltens von Mitgliedern des Unternehmens oder externer Partner [3].

Doch welchen Stellenwert nimmt die Kundenzufriedenheit im Zielsystem von Unternehmen tatsächlich ein? Diese Frage wird im Folgenden näher betrachtet und beantwortet.

\section{Kundenzufriedenheit - wesentlicher Faktor zur Existenzsicherung der Unternehmen}

Bevor der aktuelle Stand zu diesem Thema untersucht wird, soll zunächst einmal eine gültige Definition von Kundenzufriedenheit gefunden werden. In der einschlägigen Fachliteratur finden sich derlei viele - eine mögliche davon, ist die nach C. Handy [4]. Er definierte Kundenzufriedenheit als

„The gap of distance between consumer's ideal attribute combination for a particular product or service and the attribute combination of the product or service offered in the marketplace which comes closest to this ideal."

\footnotetext{
${ }^{1}$ Dipl.-Betriebswirt Thomas Bader, FIDUCIA IT AG, Karlsruhe (Germany), Thomas.Bader@Fiducia.de ${ }^{2}$ Assoc. Prof. Iveta Kremenova,PhD., University of Zilina, Faculty of Operation and Economics of Transport and Communications, Department of Communications, Iveta.Kremenova@fpedas.utc.sk
} 
Verfolgt man nun die Diskussion um den Erhalt bzw. die Steigerung der Wettbewerbsfähigkeit eines Unternehmens vor dem Hintergrund eines sich permanent verschärfenden dynamischen Wettbewerbs sowie weiterer Einflussgrößen, die aus fundamentalen Veränderungen der politisch-gesellschaftlichen, technologischen, ökologischen und ökonomischen Systeme resultieren, wird deutlich, dass innerhalb der vergangenen Jahre insbesondere die Variablen Markt- und Kundenorientierung als entscheidende Einflussfaktoren im Wettbewerb angesehen werden. Das Konstrukt Kundenzufriedenheit wird dabei als Maßstab einer kundenorientierten Unternehmensführung herangezogen.

Viele der in diesem Zusammenhang aktuell diskutierten Managementkonzepte, die auf dem Kontext eines ganzheitlichen am Kunden ausgerichteten Qualitätskonzeptes basieren, orientieren sich an der Zufriedenheit der Kunden als oberste Verhaltensmaxime und betrachten die anzustrebende Steigerung der Produkt- und / oder Dienstleistungsqualität sowie der entsprechenden Kundenzufriedenheit nicht nur als eine funktionale Teilverantwortung, sondern vielmehr als eine unternehmerische Führungsaufgabe [6].

Die nachfolgende Abbildung stellt in diesem Kontext die individuellen Kundenreaktionen in Abhängigkeit von der Richtung des Zufriedenheitsurteils dar und unterstreicht / verdeutlicht damit nochmals die Wichtigkeit zufriedene Kunden zu erlangen [7].

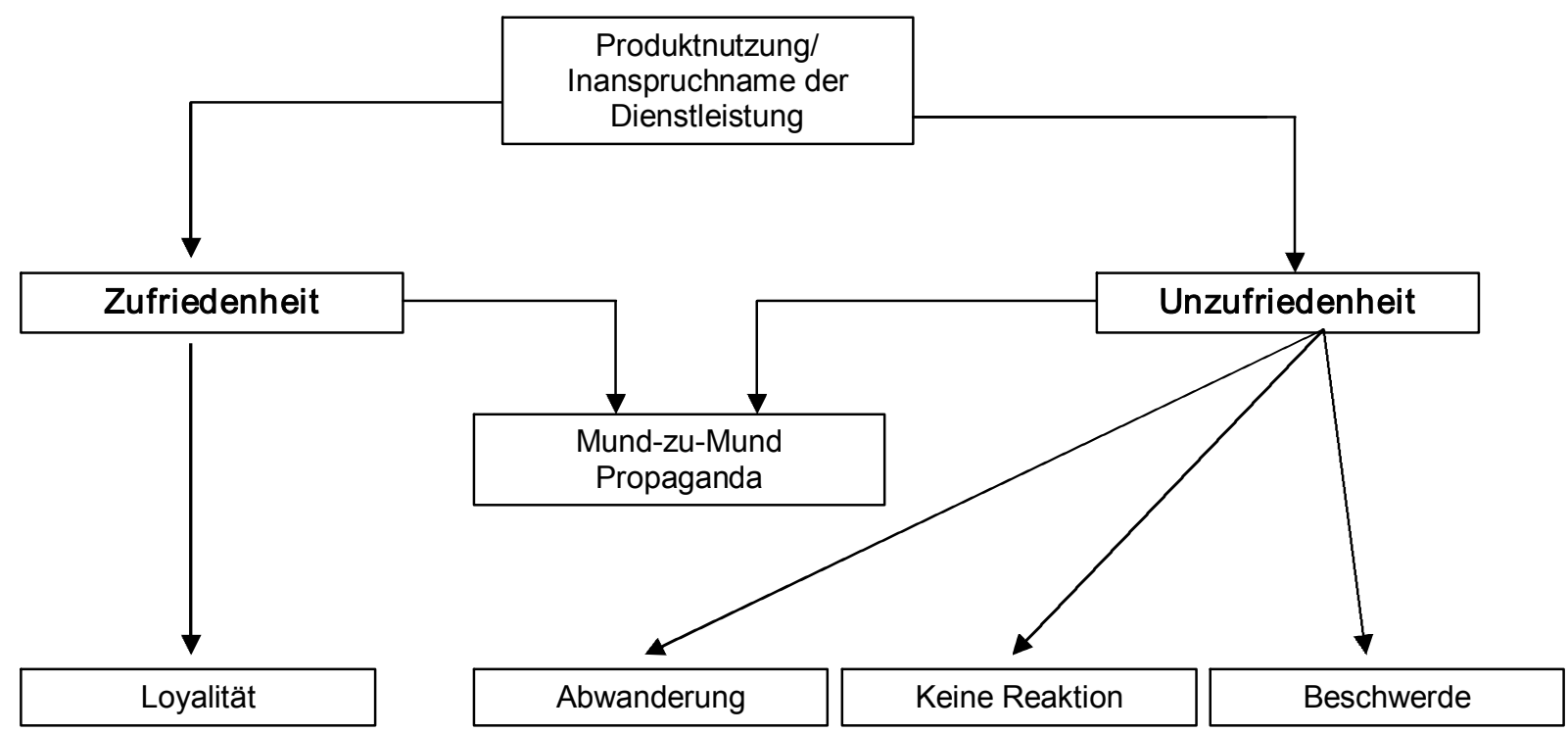

\section{Abb. 1: Individuelle Kundenreaktionsformen in Abhängigkeit von der Richtung des Zufriedenheitsurteils}

Eine Studie aus dem Jahre 2006 der Service Barometer AG in München, beschäftigte sich ebenfalls mit dem Thema Kundenzufriedenheit. Dabei ging es bspw. um Fragestellungen wie:

- Wie werden Kundenzufriedenheitsmessungen vorgenommen?

- Wie werden die Ergebnisse aus Zufriedenheitsbefragungen für konkrete Managementzwecke genutzt? 
Folgende Erkenntnisse konnten aus dem Ergebnis der Studie abgeleitet werden:

- Das Handlungsfeld Kundenzufriedenheit wird auch noch heute häufig primär als Messproblem angesehen, während die damit verbundenen Managementherausforderungen nicht im Fokus der Betrachtung standen.

- Verantwortlich für die Kundenzufriedenheit im Unternehmen sind vielfach das Marketing bzw. die Marktforschung. D.h. eine Funktion, die traditionell zwar die Kundenperspektive einnimmt, aber wenig Einfluss auf weitergehende Managemententscheidungen hat.

- Kundenzufriedenheit wird zwar als wichtiges Unternehmensziel proklamiert, aber nur in begrenztem Umfang tatsächlich als Zielgröße konkret definiert und in Zielvereinbarungen für Manager und Mitarbeiter berücksichtigt.

Das bedeutet, dass eine Änderung erst dann zu erwarten ist, wenn Zufriedenheitsmanagement in der Unternehmensleitung verankert und Kundenzufriedenheit als strategische Größe im Zielsystem fixiert und für konkrete Prozesse heruntergebrochen wird [3].

Wie die Ergebnisse der dargestellten Studie belegen, wird aktuell dem Thema Kundenzufriedenheit noch immer nicht die entsprechende Gewichtung zugeschrieben.

Allerdings ist derzeit zu beobachten, dass die Unternehmen (wenngleich auch noch nicht alle) beginnen, sich mit diesem Thema verstärkt auseinanderzusetzen, da sie die Notwendigkeit dafür erkannt haben.

Auf Grund der geschilderten Situation aber, sollte jedes Unternehmen - neben der Formulierung seiner bisherigen Unternehmensziele wie bspw. Gewinnsteigerung um einen bestimmten Prozentsatz oder Übernahme der technologischen Führerschaft - auch Kundezufriedenheit als weiteres Unternehmensziel aufnehmen.

Damit das Unternehmensziel „Kundenzufriedenheit“ nicht nur ein Lippenbekenntnis bleibt, sondern aktiv im Unternehmen gelebt und im Besonderen von der Unternehmensleitung vorgelebt wird, ist es notwendig, es mit einem entsprechenden Messund Controllingsystem zu unterlegen, denn Ziele müssen operationalisierbar sein.

Dies könnte bspw. in der Form erfolgen, dass die Höhe der Prämien- und Tantiemenzahlungen an die Mitarbeiter und Führungskräfte vom Ergebnis durchgeführter Kundenumfragen (verbesserte oder verschlechterte sich die Kundenzufriedenheit) abhängig gemacht werden würden. $\mathrm{Zu}$ beachten wäre dabei, dass die Umfrage von einem externen, unabhängigen Institut durchgeführt werden würde, um die Objektivität zu wahren.

Als Beispiel für eine gelungene Umsetzung des Themas Kundenzufriedenheit als Unternehmensziel steht das weltweit tätige Chemieunternehmen BASF. Dieses Unternehmen definiert in seinen im Internet [1] veröffentlichte Leitlinien unter anderem:

„Wir entwickeln und optimieren unsere Produkte und Dienstleistungen gemeinsam mit unseren Kunden so, dass wir zur Wertsteigerung bei unseren Kunden und in unserem Unternehmen beitragen."

„Wir messen regelmäßig die Kundenzufriedenheit. Hinweise unserer Kunden und Partner nutzen wir konsequent zur Verbesserung unserer Geschäftsprozesse. “ 
Dass sich das aktive Management von Kundenzufriedenheit auch wirtschaftlich lohnt, wird von den Unternehmen, die dies als Unternehmensziel definiert haben, bescheinigt.

Egal ob es sich dabei um große oder kleine Unternehmen handelt, sie alle agieren damit ausgesprochen erfolgreich am Markt - immer jedoch unter der Voraussetzung, dass Produktund / oder Dienstleistungsqualität stimmen.

Auch in der Fachliteratur werden diese ökonomischen Vorteile bspw. von Rosenstiel und Neumann [9] bestätigt. Denn es ist weitaus billiger mit einem zufriedenen Kunden ein Folgegeschäft abzuschließen, als einen Neu-Kunden zu gewinnen bzw. einen enttäuschten Kunden zu versöhnen. Darüber hinaus ergeben sich in diesem Zusammenhang außerdem noch weitere Kostenvorteile in der Form, dass man bei zufriedenen Kunden einer einmal eingeschlagenen Unternehmensstrategie treu bleiben und so vergleichbare Konzepte auch in Zukunft nutzen kann.

\section{Ergebnis}

Nur wenn es gelingt Kundenzufriedenheit als Unternehmensziel - in Verbindung mit den entsprechenden Mess- und Controllinginstrumenten - zu etablieren, werden die Bemühungen der Unternehmen, die Kundenzufriedenheit kontinuierlich $\mathrm{zu}$ verbessern, erfolgreich sein.

Auf der Suche nach unterstützenden Mitteln zum Erreichen von langfristiger Kundenzufriedenheit, stößt man dabei unweigerlich auf Qualitätsmanagementsysteme (QMSysteme).

Unter dem Begriff Qualitätsmanagementsystem versteht man ein System zur Festlegung der Qualitätspolitik und der Qualitätsziele sowie des Erreichens dieser Ziele.

Ein Qualitätsmanagementsystem ist die Gesamtheit der aufbau- und ablauforganisatorischen Gestaltung, sowohl zur Verknüpfung der qualitätsbezogenen Aktivitäten untereinander wie auch im Hinblick auf eine einheitliche, gezielte Planung, Umsetzung und Steuerung der Maßnahmen des Qualitätsmanagements im Unternehmen. Dabei wird nicht nur die Produktion mit ihren vor- und nachgelagerten Bereichen einbezogen, sondern das gesamte Unternehmen einschließlich der Beziehungen zu seinem Umfeld. Es entsteht ein System vernetzter Regelkreis auf allen betrieblichen Ebenen, wodurch Ziele, Struktur, Verantwortlichkeiten, Verfahren, Prozesse und die zur Durchführung erforderlichen Mittel festgelegt werden. Das Qualitätsmanagementsystem dient somit der Strukturierung und der systematischen Umsetzung von Qualitätsaufgaben im Unternehmen [8].

Eines der dabei am weitest verbreiteten Qualitätsmanagementsysteme ist die Normenfamilie ISO 9000:2000 ff., deren Stärken liegen neben den oben definierten Aufgaben besonders im Bereich der Verbesserung der Kundenzufriedenheit.

Mittlerweile nutzen bereits mehr als 700.000 Unternehmen in über 160 Ländern erfolgreich dieses QM-System bzw. haben sich nach der Einzelnorm ISO 9001:2000 zertifizieren lassen [5]. 


\section{Literaturverzeichnis}

[1] BASF: Innovation für den Erfolg unserer Kunden, Vision-Grundwerte-Leitlinien, 2007, S. 1, http://www.corporate .basf.com/de

[2] CEN Europäisches Komitee für Normung: Qualitätsmanagementsysteme Grundlagen und Begriffe (ISO 9000:2000), in Management-Zentrum Brüssel, Brüssel 2000, S. 6 f

[3] COENEN. C. ; STAUSS, B.: Kundenzufriedenheitsmanagement in Deutschland - Nicht nur drüber reden!, in QZ, Jahrgang 51, Nr. 9, Carl Hanser Verlag, München 2006, S. 22 ff

[4] HANDY, C. R.: Monitoring Consumer Satisfaction with Food Products, in Conceptualization and Measurement of Consumer Satisfaction and Dissatisfaction, Proceedings of Conference Conducted by Marketing Science Institute. Hrsg. Hunt, H.K., Cambridge/Mass. 1977, S. 217

[5] ISO: The ISO Survey sheds light on roles of management system standards in globalization, ISO Press releases Ref.: 1021, August 2006, S. 1, www. iso.org/iso/en/commcentre/ pressreleases/2006/Ref1021.html?prtable=true

[6] KAISER, M.-O.: Erfolgsfaktor Kundenzufriedenheit, Erich Schmidt Verlag, Berlin 2005 a, S. 8 f, ISBN 3-503-07833-9

[7] KAISER, M.-O.: Erfolgsfaktor Kundenzufriedenheit, Erich Schmidt Verlag, Berlin 2005 b, S. 29, ISBN 3-503-07833-9

[8] KAMISKE, G. F.; BRAUER, J.-P.: Qualitätsmanagement von A - Z, Carl Hanser Verlag, München Wien 2006, S. 210 f, ISBN 3-446-40284-5

[9] ROSENSTIEL VON L.; NEUMANN, P.: Mehr als ein Käufer - Der Kunde, das unbekannte Wesen, in Künzel, H. (Hrsg.), Handbuch Kundenzufriedenheit, Springer-Verlag Berlin Heidelberg 2005 , S. 25, ISBN 3-540-21144-6 